\title{
Comparison of algorithms for computing infinite structural indices of polynomial matrices
}

\author{
Juan-Carlos Zúñiga ${ }^{1}$, Didier Henrion ${ }^{1,2,3}$
}

November 27, 2002

\section{Keywords}

Linear systems, polynomial matrices, structural indices, decoupling control, numerical linear algebra, computer-aided control system design.

\begin{abstract}
A new algorithm is proposed to compute the infinite structural indices of a polynomial matrix, i.e. the algebraic and geometric multiplicities of its poles and zeros at infinity. Infinite structural indices of polynomial matrices play a key role when solving e.g. multivariable decoupling control problems. The algorithm is based on numerically stable operations only, and takes full advantage of the block Toeplitz structure of a constant matrix built directly from the polynomial matrix coefficients. Comparative numerical examples and a full computational complexity analysis indicate that the Toeplitz algorithm can be viewed as a competitive alternative to the well-known state-space pencil matrix algorithm for obtaining structural indices.
\end{abstract}

\section{Introduction}

Polynomials and polynomial matrices arise naturally and cannot be avoided in linear system theory. By analogy with scalar rational transfer functions, matrix transfer functions of linear systems can be written in polynomial matrix fraction form as $N(s) D^{-1}(s)$ where $D(s)$ is a non-singular polynomial matrix $[8,6,1]$.

Zeros of polynomial matrices naturally represent either poles or zeros of linear multivariable systems described by polyno-

\footnotetext{
${ }^{1}$ Laboratoire d'Analyse et d'Architecture des Systèmes, Centre National de la Recherche Scientifique. 7 Avenue du Colonel Roche, 31077 Toulouse, cedex 4, France.

${ }^{2}$ Institute of Information Theory and Automation, Academy of Sciences of the Czech Republic. Pod vodárenskou věží 4, 18208 Prague, Czech Republic.

${ }^{3}$ Corresponding author. FAX: +33 5613369 69. E-mail: henrion@laas.fr
}

mial matrix fractions. They are frequently encountered when analyzing and/or designing linear systems or filters. Associated with the zeros are the finite and infinite structures of a polynomial matrix, defined from specific canonical forms under matrix equivalence:

- The finite structure of a polynomial matrix $A(s)$ is displayed in its Smith form, where the finite zeros of $A(s)$ are the roots of the invariant polynomials appearing on the diagonal of the Smith form of $A(s)$ [3].

- The infinite structure of $A(s)$ is defined via its SmithMacMillan form at infinity, which can be regarded as a canonical form considering equivalence through biproper matrices. As part of its infinite structure, any polynomial matrix may have a certain number of infinite poles and infinite zeros of different algebraic and geometric multiplicities, or orders. [15].

In this paper, we are concerned with numerical algorithms for computing the so-called infinite structural indices of a polynomial matrix, i.e. algebraic and geometric multiplicities of its zeros and poles at infinity. Dynamically, infinite zero orders of the system are related to the number of times that the outputs of the system have to be differentiated for a component of the input vector to appear explicitly. Thus, this information is crucial in the solution of important control problems. For instance, a linear multivariable system is row by row decouplable by static state feedback if and only if the sum of its infinite zero orders equals the sum of its row infinite zero orders. Other typical control problems where infinite structural indices of polynomial matrices play a fundamental role are model matching and disturbance rejection, see the introduction in [5] and the references therein.

Up to our knowledge there basically exist two algorithms to compute structural indices of polynomial matrices:

- The pencil algorithm, based on generalized state-space realizations and the correspondence between the SmithMacMillan form of a rational matrix and the Kronecker canonical form of an associated pencil matrix [13]. The pencil algorithm allows to compute all the structural indices (finite and infinite) of a polynomial matrix [14].

- The Toeplitz algorithm, proposed in [5] as an extension of a method originally described in [4] to compute and 
extract the finite structure of a polynomial matrix. The algorithm is rather similar in spirit to the algorithm proposed in [12]. In this reference, the structure of a rational matrix at any point is computed via orthogonal transformations on Toeplitz matrices built from the Laurent expansion.

In this paper, our objective is to revisit the Toeplitz algorithm of [5] in order to take full advantage of the block Toeplitz structure and therefore to reduce the computational burden as much as possible. The algorithm is designed while keeping with our main impetus, which is the development of reliable numerical methods for dealing with polynomial matrices and their implementation in a user-friendly commercial Matlab package called the Polynomial Toolbox, developed and produced by the start-up PolyX Ltd. [10]. In this regard, all the routines used in the algorithm are numerically stable in the sense that they are based on backward stable (orthogonal) transformations [2, 9]. The Toeplitz algorithm can thus be viewed as a "polynomial approach" alternative to the "statespace approach" algorithms proposed in [13] and recently implemented in the Fortran library Slicot [11].

By a thorough computational complexity analysis, we show that the Toeplitz algorithm may be considered as a competitive alternative to the pencil algorithm for computing infinite structural indices of polynomial matrices. Several numerical examples and intensive testings illustrate the pros and cons of both algorithms.

\section{Toeplitz Approach}

Consider a non-singular $n \times n$ polynomial matrix

$$
A(s)=A_{0}+A_{1} s+\cdots+A_{d-1} s^{d-1}+A_{d} s^{d}
$$

of degree $d$ and let $T_{k}=A_{d+1-k}$.

The algorithm to obtain the structure at infinity of $A(s)$ processes the block Toeplitz matrix

$$
T=\left[\begin{array}{cccc}
T_{1} & & & \\
T_{2} & T_{1} & & \\
\vdots & \vdots & \ddots & \\
T_{k} & T_{k-1} & \cdots & T_{1}
\end{array}\right]
$$

and is described as follows:

Step 1 Obtain the singular value decomposition (SVD) $S=$ $P^{T} T_{1} Q_{1}$ and the rank $r_{1}$ of $T_{1}$.

If $v_{1}=n-r_{1}$ is not zero, make the column compression $T_{1} Q_{1}=\left[\begin{array}{ll}C_{1} & 0\end{array}\right]$ where $C_{1}$ has $r_{1}$ columns and update the next row block of $T, \widehat{T}_{2}=\left[\begin{array}{ll}T_{2} \bar{Q}_{1} T_{1}\end{array}\right]$ where $\bar{Q}_{1}$ contains the $v_{1}$ rightmost columns of $Q_{1}$.

If $v_{1}$ is zero then go to End.
Step k Obtain the SVD $S=P^{T} \widehat{T}_{k} \widehat{Q}_{k}$ and the rank $r_{k}$ of $\widehat{T}_{k}$. If $v_{k}=n-r_{k}$ is not zero, make the column compression $\widehat{T}_{k} \widehat{Q}_{k}=\left[C_{k} 0\right]$ where $C_{k}$ has $r_{k}$ columns, let

$$
Q_{k}=\left[\begin{array}{cc}
Q_{k-1} & 0 \\
0 & I_{n}
\end{array}\right]\left[\begin{array}{cc}
I_{r_{1}+r_{2}+\cdots+r_{k-1}} & 0 \\
0 & \widehat{Q}_{k}
\end{array}\right]
$$

and update the next row block of $T, \widehat{T}_{k+1}=\left[\left[T_{k+1} \cdots T_{3}\right.\right.$ $\left.\left.T_{2}\right] \bar{Q}_{k} T_{1}\right]$ where $\bar{Q}_{k}$ contains the $w_{k}=v_{1}+v_{2}+\cdots+v_{k}$ rightmost columns of $Q_{k}$.

If $v_{k}$ is zero then go to End.

End For $i=1,2, \ldots, k-1$, if $i \geq d$ then $A(s)$ has $v_{i}-v_{i+1}$ zeros at infinity of degree $i-d$. If $i \leq d$ then $A(s)$ has $v_{i}-v_{i+1}$ poles at infinity of degree $d-i$. $A(s)$ has $n-v_{1}$ poles at infinity of degree $d$.

In order to count the number of operations that the previous algorithm requires, we present its basic code in a Matlab-like syntax.

\section{Function sinftoep}

Returns vector $v=\left[\begin{array}{lll}v_{1} & v_{2} & \cdots\end{array}\right]$ of nullities characterizing the structure at infinity of a given non-singular polynomial matrix $A(s) \in \mathbb{R}^{n \times n}[s]$ of degree $d$.

$$
\begin{array}{ll}
\text { Step } 0 & \widehat{T}_{1}=T_{1} \\
& w_{0}=0 \\
& Q_{0}=I_{n} \\
& k=1
\end{array}
$$

Step k $\left[P, D, \widehat{Q}_{k}\right]=\operatorname{svd}\left(\widehat{T}_{k}\right)$

$$
\begin{aligned}
& r_{k}=\operatorname{rank}\left(\widehat{T}_{k}\right) \\
& v_{k}=n-r_{k} \\
& w_{k}=w_{k-1}+v_{k} \\
& \text { If } v_{k}=0, \text { goto End } \\
& Q_{k_{1}}=Q_{k-1} * \widehat{Q}_{k}\left(1: w_{k-1},\left(r_{k}+1\right)\right. \\
& Q_{k_{2}}=\widehat{Q}_{k}\left(\left(w_{k-1}+1\right):\left(n+w_{k-1}\right)\right. \\
& Q_{k}=\left[Q_{k_{1}} ; Q_{k_{2}}\right] \\
& \widehat{T}_{k+1}=\left[\left[T_{k+1} \cdots T_{3} T_{2}\right] * Q_{k} T_{1}\right] \\
& k=k+1
\end{aligned}
$$$$
Q_{k_{1}}=Q_{k-1} * \widehat{Q}_{k}\left(1: w_{k-1},\left(r_{k}+1\right):\left(n+w_{k-1}\right)\right)
$$$$
Q_{k_{2}}=\widehat{Q}_{k}\left(\left(w_{k-1}+1\right):\left(n+w_{k-1}\right),\left(r_{k}+1\right):\left(n+w_{k-1}\right)\right)
$$

End Return $v$

We consider that an SVD for a matrix $A \in \mathbb{R}^{m \times n}$ requires $4 m^{2} n+8 m n^{2}+9 n^{3}$ operations, and that a multiplication $C=$ $A B$ with $A \in \mathbb{R}^{m_{A} \times n}$ and $B \in \mathbb{R}^{n \times n_{B}}$ requires $(2 n-1) m_{A} n_{B}$ operations [2].

In step $\mathrm{k}$, sinf toep executes one SVD of dimension $n \times(n+$ $\left.w_{k-1}\right)$, one multiplication of dimensions $(k-1) n \times w_{k-1}$ by $w_{k-1} \times w_{k}$, and one multiplication of dimensions $n \times k n$ by $k n \times w_{k}$, namely

$$
\begin{array}{r}
\text { flops }_{T_{k}}=9 w_{k-1}^{3}+n(33+2 k) w_{k-1}^{2}+ \\
n\left(47 n+2 k n+2 v_{k} k-2 v_{k}-k\right) w_{k-1}+ \\
k n(2 n-1) v_{k}+21 n^{3}
\end{array}
$$

operations. 
Supposing that $q$ steps are needed to recover all the structure at infinity of matrix $A(s)$, that is to say $v_{q+1}=0$. The total number of operations that sinftoep performs is equal to the sum of the number of operations in the $q$ steps plus flops ${ }_{T_{q+1}}=$ $9 w_{q}^{3}+35 n w_{q}^{2}+47 n^{2} w_{q}+21 n^{3}$ that is the number of operations needed to compute the last SVD from which we obtain $v_{q+1}=$ 0, i.e.

$$
\text { flops }_{T}=\sum_{k=1}^{q+1} \text { flops }_{T_{k}}
$$

\section{Pencil Approach}

The algorithm to obtain the structure at infinity of the matrix $A(s)$ given in (1) processes the pencil

$$
\begin{aligned}
P(s)=s E-L=s & {\left[\begin{array}{lllll} 
& -I_{n} & & \\
& & \ddots & \\
& & & -I_{n} \\
& & & & \\
A_{0} & A_{1} & \cdots & A_{d-1}
\end{array}\right]-} \\
& {\left[\begin{array}{ccccc}
-I_{n} & & & \\
& \ddots & & \\
& & -I_{n} & \\
& & & -A_{d}
\end{array}\right] }
\end{aligned}
$$

and is described as follows. For more details see [13].

Let us take $L_{1}=L, E_{1}=E, w_{0}=0$, and $r_{0}=n d$.

Step k Obtain the SVD $S=P^{T} L_{k} Q$ and the rank $r_{k}$ of $L_{k}$. If $v_{k}=r_{k-1}-r_{k}$ is not zero, make the column compressions $L_{k} Q=\left[R_{L_{k}} 0\right]$, and $E_{k} Q=\left[R_{E_{k}} N_{k}\right]$, where $R_{E_{k}}$ and $R_{L_{k}}$ have $r_{k}$ columns. Obtain the SVD $S=P^{T} N_{k} Q$, and make the permuted row compressions

$$
\begin{array}{r}
I_{p} P^{T} R_{k}=\left[\begin{array}{c}
L_{k+1} \\
R_{L_{k, 2}}
\end{array}\right], I_{p} P^{T} R b_{k}=\left[\begin{array}{c}
E_{k+1} \\
R_{E_{k, 2}}
\end{array}\right], \\
I_{p} P^{T} N_{k}=\left[\begin{array}{c}
0 \\
N_{k, 2}
\end{array}\right]
\end{array}
$$

where $L_{k+1}$ and $E_{k+1}$ have $r_{k}$ rows, and

$$
I_{p}=\left[\begin{array}{cc}
0 & I_{r_{k}} \\
I_{v_{k}} & 0
\end{array}\right]
$$

If $v_{k}$ is zero then go to End.

End For $i=1,2, \ldots, k-1$, if $i \geq d$ then $A(s)$ has $v_{i}-v_{i+1}$ zeros at infinity of degree $i-d$. If $i \leq d$ then $A(s)$ has $v_{i}-v_{i+1}$ poles at infinity of degree $d-i$. $A(s)$ has $n-v_{1}$ poles at infinity of degree $d$.

In order to count the number of operations that the previous algorithm requires, we also present its basic code in a Matlablike syntax.

\section{Function sinfpenc}

Returns vector $v=\left[\begin{array}{lll}v_{1} & v_{2} & \cdots\end{array}\right]$ of nullities characterizing the structure at infinity of a given non-singular polynomial matrix $A(s) \in \mathbb{R}^{n \times n}[s]$ of degree $d$.

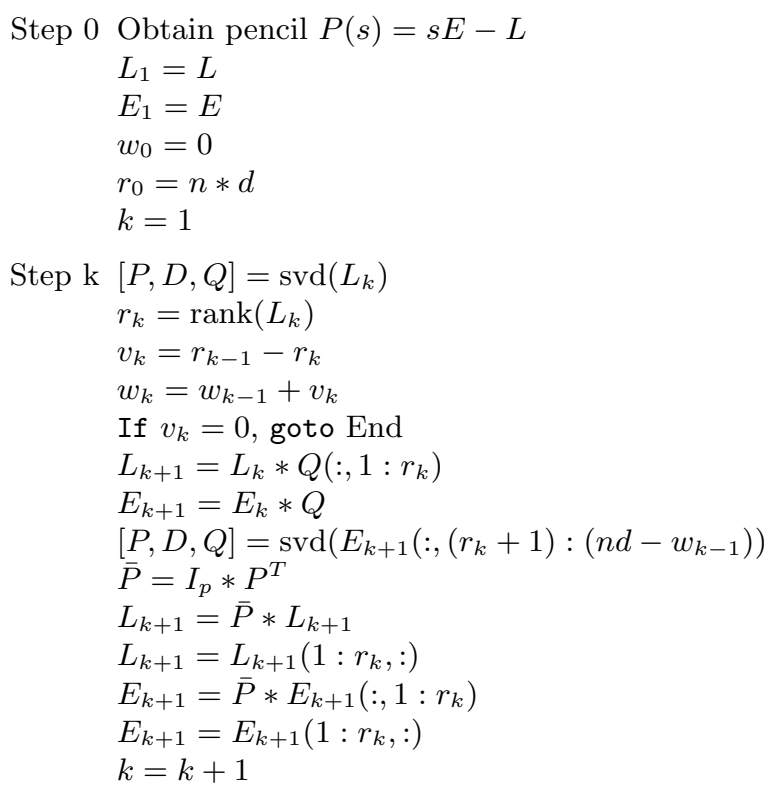

End Return $v$

In step $\mathrm{k}$, sinfpenc executes one SVD of dimension ( $n d-$ $\left.w_{k-1}\right) \times\left(n d-w_{k-1}\right)$, another one of dimension $\left(n d-w_{k-1}\right) \times v_{k}$, two multiplications of dimensions $\left(n d-w_{k-1}\right) \times\left(n d-w_{k-1}\right)$ by $\left(n d-w_{k-1}\right) \times\left(n d-w_{k-1}\right)$, and three multiplications of dimensions $\left(n d-w_{k-1}\right) \times\left(n d-w_{k-1}\right)$ by $\left(n d-w_{k-1}\right) \times(n d-$ $\left.w_{k}\right)$, namely

$$
\begin{gathered}
\text { flops }_{P_{k}}=-31 w_{k-1}^{3}+\left(93 n d-2 v_{k}-5\right) w_{k-1}^{2}+ \\
\left(10 n d+4 n d v_{k}-93 n^{2} d^{2}-3 v_{k}-8 v_{k}^{2}\right) w_{k-1}+ \\
9 v_{k}^{3}+8 n d v_{k}^{2}+n d(3-2 n d) v_{k}+n^{2} d^{2}(31 n d-5)
\end{gathered}
$$

operations

Now, supposing that $q$ steps are needed to recover all the structure at infinity of matrix $A(s)$, and considering that flops $_{P_{q+1}}=-21 w_{q}^{3}+63 n d w_{q}^{2}-63 n^{2} d^{2} w_{q}+21 n^{3} d^{3}$ operations are needed to compute the last SVD from which we obtain $v_{q+1}=0$, we get that the total number of operations that sinftoep performs is equal to

$$
\text { flops }_{P}=\sum_{k=1}^{q+1} \text { flops }_{P_{k}}
$$

\section{Comparison}

As we can see from equations (2) and (4), when obtaining the structure at infinity of a polynomial matrix $A(s)$ several quantities are involved, not only the dimension $n$ of the matrix, but also its degree $d$ and in general its structure, namely nullities $v_{k}$ and number of steps $q$. 
In this section we present an analysis of the dependence of the functions sinfpenc and sinftoep on these dimensions. First we consider that the nullities and the number of steps are fixed and we analyze the dependence on $n$ and $d$. Then, we consider the general case, namely, when nullities and number of steps are not fixed.

\subsection{Dependence on the degree $d$ and the dimension $n$ for given infinite struc- tural indices}

If we consider the nullities fixed, we can see from equation (4) that the algorithmic complexity of the pencil approach is $O\left(n^{3} d^{3}\right)$. On the other hand, with the Toeplitz approach we can eliminate the dependence on degree $d$. We can see from equation (2) that the algorithmic complexity is $O\left(n^{3}\right)$. The reason is that the pencil algorithm always executes an SVD of dimension $n d \times n d$.

Test 1 Consider the polynomial matrix of degree $d$

$$
A(s)=\left[\begin{array}{ccc}
s^{d} & p_{1}(s) & p_{2}(s) \\
0 & s^{d-a} & p_{3}(s) \\
0 & 0 & s^{d-a-b}
\end{array}\right],
$$

where $a, b$ are given integers and $p_{1}(s), p_{2}(s), p_{3}(s)$ are given polynomials.

If the degree of $p_{3}(s)$ is less than $d-a$, then we can see that the vector of the nullities of $A(s)$ is

$$
v=[\underbrace{2,2, \ldots, 2}_{a}, \underbrace{1,1, \ldots, 1}_{b}, 0] .
$$

Now fix the structure of $A(s)$ with $a=5$ and $b=2$ and vary the degree $d$ in order to show how it affects the number of operations in functions sinftoep and sinfpenc. The results are presented in the table below.

\begin{tabular}{|r|r|r|}
\hline Matrix degree & flops $_{T}$ & flops $_{P}$ \\
\hline \hline 20 & 136683 & 37181275 \\
\hline 40 & 136683 & 349798015 \\
\hline 60 & 136683 & 1246161955 \\
\hline 80 & 136683 & 3034721095 \\
\hline
\end{tabular}

We can see that the algorithmic complexity of function sinftoep does not depend directly on the degree of the matrix. On the other hand, we can see the rapid growth in the number of operations executed by function sinfpenc when degree $d$ increases.

Test 2 Now consider the polynomial matrix

$$
A(s)=\left[\begin{array}{cccc}
s^{3} I_{b} & & & \\
& 1 & s^{3} & 0 \\
& 0 & 1 & s \\
& 0 & 0 & 1
\end{array}\right]
$$

where $b$ is a given integer.

For this matrix, we can increase $b$ in order to increase the dimension $n$ of $A(s)$, nevertheless the degree $d=3$ and the vector of nullities $v$ do not change.

We are going to vary $b$ in order to show how it affects the number of operations in functions sinftoep and sinfpenc. The results are presented in the table below.

\begin{tabular}{|r|r|r|}
\hline Matrix dimension & flops $_{T}$ & flops $_{P}$ \\
\hline \hline 20 & 2518093 & 39830120 \\
\hline 40 & 14935813 & 362273240 \\
\hline 60 & 45335133 & 1275721160 \\
\hline 80 & 101780053 & 3088621880 \\
\hline
\end{tabular}

Now we can see that algorithmic complexities of both functions depend on the dimension $n$.

From these results we can conclude that one apparent advantage of Toeplitz approach is the independence on the matrix degree in some specific cases. Nevertheless, generally a change in the degree or the dimension of a polynomial matrix causes a change in its structure at infinity, namely a change in the values of nullities $v_{k}$ and in the number of steps $q$. Then, in order to determine which approach is more efficient, we are going to analyze equations (3) and (5) in a more general way.

\subsection{General case, dependence on infinite structural indices}

First, we have to bound numbers $q, v_{k}$ and $w_{k}$ for $k=$ $1,2, \ldots, q$. Let us consider a non-singular polynomial matrix $A(s) \in \mathbb{R}^{n \times n}[s]$ of degree $d$. From the Toeplitz approach it is easy to see that

$$
\begin{array}{r}
n-1 \geq v_{1} \geq v_{2} \geq v_{3} \geq \cdots \geq v_{q} \geq 1, \\
k \leq w_{k} \leq k(n-1) .
\end{array}
$$

However, from the pencil approach we know that $w_{k}$ cannot be greater than $n d$. Said in another way, when $w_{k}=n d$ we have all the structure at infinity of $A(s)$, then in general $k \leq w_{k} \leq n d$.

From this results we conclude that in the worst case, when $v_{1}=v_{2}=\cdots=v_{q}=1$, at most $q=n d$ steps are required to obtain the structure at infinity of $A(s)$, namely

$$
1 \leq q \leq n d
$$

As to the number of operations, it can be seen that the worst case for the pencil approach is when $v_{k}$ is small. $v_{k}$ small implies not only more steps, but also operations on larger matrices because the deflation at each step will be smaller. On the other hand, when $v_{k}$ is large, the number of steps and the dimension of the operations are reduced. It is more 
difficult to find the worst or the best case for the Toeplitz approach. If $v_{k}$ is small we have less steps, however we also have a smaller deflation, namely operations on larger matrices. On the other hand, if $v_{k}$ is large the number of steps increases but the deflation increases too, namely, smaller matrices are processed.

To continue with this analysis we consider the extreme cases, when $v_{k}=1$ and when $v_{k}=n-1$ and we compare the number of operations performed by functions fuctions sinftoep and sinfpenc. Finally we give a general conclusion.

\section{Extreme case 1: $v_{k}=n-1$}

First let us consider that $v_{k}=n-1$ for $k=1,2, \ldots, q$. We know that at the last step $w_{q-1}=(q-1)(n-1) \leq n d$, therefore the maximum number of steps is $q_{\max }=$ floor $\left(\frac{n d}{n-1}+1\right)$, where floor $(x)$ is the largest integer less than or equal to $x$. However, it is important to notice that in a non-singular polynomial matrix the number of poles (we only have poles at infinity) must be equal to the number of zeros (finite or infinite) including multiplicities [15]. This condition limits the maximum number of steps $q_{\max }$. For example consider a polynomial matrix $A(s)$ of degree 3 and dimension 7 , if $v_{k}=n-1=6$, $A(s)$ has one pole at infinity of degree 3 and we can not have $q=$ floor $\left(\frac{n d}{n-1}+1\right)=4$ because this would imply that $A(s)$ has 6 zeros at infinity of degree 1 , hence 3 finite poles which is impossible. Then, in general we can verify that $q_{\max }=d$.

Test 3 Consider an $n \times n$ polynomial matrix of degree $d$, with nullities $v_{1}=v_{2}=\cdots=v_{q}=n-1$.

In the following tables for various values of $n, d$ and $q$, we denote with the plus sign $(+)$ problem instances for which the Toeplitz algorithm executes less operations than the pencil algorithm, and with the minus sign (-) problem instances for which the Toeplitz algorithm executes more operations.

\begin{tabular}{|c|c|c|c|c|c|c|c|c|c|c|c|c|c|}
\hline$q \backslash n$ & 3 & 4 & 5 & 6 & 7 & 8 & 9 & 10 & 11 & 12 & 13 & 14 & 15 \\
\hline 1 & - & - & - & - & - & - & - & - & - & - & - & - & - \\
\hline
\end{tabular}

Table 1. $d=1, v_{k}=n-1$

We can see from Table 1 that when $d=1$ the pencil algorithm is always more efficient. The reason is that in step $q+1$, while the pencil algorithm processes a matrix of dimension $1 \times 1$, the Toeplitz algorithm processes a matrix of dimension $n \times(2 n+1)$.

\begin{tabular}{|c|c|c|c|c|c|c|c|c|c|c|c|c|c|}
\hline$q \backslash n$ & 3 & 4 & 5 & 6 & 7 & 8 & 9 & 10 & 11 & 12 & 13 & 14 & 15 \\
\hline 1 & + & + & + & + & + & + & + & + & + & + & + & + & + \\
\hline 2 & + & + & - & - & - & - & - & - & - & - & - & - & - \\
\hline
\end{tabular}

Table 2. $d=2, v_{k}=n-1$
From Table 2 we can see that when degree $d$ increases (in this table $d=2$ ), q must be equal to $d$ for the pencil algorithm to be more efficient.

\begin{tabular}{|c|c|c|c|c|c|c|c|c|c|c|c|c|c|}
\hline$q \backslash n$ & 3 & 4 & 5 & 6 & 7 & 8 & 9 & 10 & 11 & 12 & 13 & 14 & 15 \\
\hline 1 & + & + & + & + & + & + & + & + & + & + & + & + & + \\
\hline 2 & + & + & + & + & + & + & + & + & + & + & + & + & + \\
\hline 3 & + & + & + & + & + & + & + & + & + & + & + & + & + \\
\hline
\end{tabular}

Table 3. $d=3, v_{k}=n-1$

When d keeps increasing, not only q must be equal to $d$ but also $n$ must be very large. For exemple, when $d=3$, the pencil algorithm is more efficient only when $n>36$.

Extreme case 2: $v_{k}=1$

Now let us consider that $v_{k}=1$ for $k=1,2, \ldots, q$. In this case we can easily verify that $q_{\max }=n d$. For example consider a polynomial matrix $A(s)$ of degree 3 and dimension 7 . If $v_{k}=1$ with $q=1,2, \ldots, n d, A(s)$ has 6 poles at infinity of degree 3 and one zero at infinity of degree 18 .

Test 4 Consider an $n \times n$ polynomial matrix of degree $d$ with nullities $v_{1}=v_{2}=\cdots=v_{q}=1$.

In the following tables, notations are the same as in Test 3 and empty cells denote impossible problem instances (when $q>n d)$.

\begin{tabular}{|c|c|c|c|c|c|c|c|c|c|c|c|c|c|}
\hline$q \backslash n$ & 3 & 4 & 5 & 6 & 7 & 8 & 9 & 10 & 11 & 12 & 13 & 14 & 15 \\
\hline $1-3$ & - & - & - & - & - & - & - & - & - & - & - & - & - \\
\hline 4 & & - & - & - & - & - & - & - & - & - & - & - & - \\
\hline 5 & & & - & - & - & - & - & - & - & - & - & - & - \\
\hline 6 & & & & - & - & - & - & - & - & - & - & - & - \\
\hline 7 & & & & & - & - & - & - & - & - & - & - & - \\
\hline 8 & & & & & & - & - & - & - & - & - & - & - \\
\hline 9 & & & & & & & - & - & - & - & - & - & - \\
\hline 10 & & & & & & & - & - & - & - & - & - \\
\hline 11 & & & & & & & & & - & - & - & - & - \\
\hline 12 & & & & & & & & - & - & - & - \\
\hline 13 & & & & & & & & & & - & - & - \\
\hline 14 & & & & & & & & & & & - & - \\
\hline 15 & & & & & & & & & & & - \\
\hline
\end{tabular}

Table 4. $d=1, v_{k}=1$

From Table 4 we can see that when $d=1$ the pencil algorithm is always more efficient. 


\begin{tabular}{|c|c|c|c|c|c|c|c|c|c|c|c|c|c|}
\hline$q \backslash n$ & 3 & 4 & 5 & 6 & 7 & 8 & 9 & 10 & 11 & 12 & 13 & 14 & 15 \\
\hline $1-7$ & + & + & + & + & + & + & + & + & + & + & + & + & + \\
\hline 8 & - & + & + & + & + & + & + & + & + & + & + & + & + \\
\hline 9 & - & + & + & + & + & + & + & + & + & + & + & + & + \\
\hline 10 & & + & + & + & + & + & + & + & + & + & + & + & + \\
\hline 11 & & - & + & + & + & + & + & + & + & + & + & + & + \\
\hline 12 & & - & + & + & + & + & + & + & + & + & + & + & + \\
\hline 13 & & & - & + & + & + & + & + & + & + & + & + & + \\
\hline 14 & & & - & + & + & + & + & + & + & + & + & + & + \\
\hline 15 & & & - & + & + & + & + & + & + & + & + & + & + \\
\hline 16 & & & & - & + & + & + & + & + & + & + & + & + \\
\hline 17 & & & & - & + & + & + & + & + & + & + & + & + \\
\hline$\vdots$ & $\vdots$ & $\vdots$ & $\vdots$ & $\vdots$ & $\vdots$ & $\vdots$ & $\vdots$ & $\vdots$ & $\vdots$ & $\vdots$ & $\vdots$ & $\vdots$ & $\vdots$ \\
\hline 45 & & & & & & & & & & & & & - \\
\hline
\end{tabular}

Table 5. $d=3, v_{k}=1$

When $d$ increases we can see that the pencil algorithm is more efficient only when $q$ approaches $q_{\max }=n d$.

\begin{tabular}{|c|c|c|c|c|c|c|c|c|c|c|c|c|c|}
\hline$q \backslash n$ & 3 & 4 & 5 & 6 & 7 & 8 & 9 & 10 & 11 & 12 & 13 & 14 & 15 \\
\hline $1-35$ & + & + & + & + & + & + & + & + & + & + & + & + & + \\
\hline 36 & & & & - & + & + & + & + & + & + & + & + & + \\
\hline 37 & & & & & + & + & + & + & + & + & + & + & + \\
\hline 38 & & & & & + & + & + & + & + & + & + & + & + \\
\hline 39 & & & & & + & + & + & + & + & + & + & + & + \\
\hline 40 & & & & & + & + & + & + & + & + & + & + & + \\
\hline 41 & & & & & - & + & + & + & + & + & + & + & + \\
\hline 42 & & & & & - & + & + & + & + & + & + & + & + \\
\hline 43 & & & & & & + & + & + & + & + & + & + & + \\
\hline 44 & & & & & & + & + & + & + & + & + & + & + \\
\hline 45 & & & & & & + & + & + & + & + & + & + & + \\
\hline$\vdots$ & $\vdots$ & $\vdots$ & $\vdots$ & $\vdots$ & $\vdots$ & $\vdots$ & $\vdots$ & $\vdots$ & $\vdots$ & $\vdots$ & $\vdots$ & $\vdots$ & $\vdots$ \\
\hline 90 & & & & & & & & & & & & & - \\
\hline
\end{tabular}

Table 6. $d=6, v_{k}=1$

When d keeps increasing, not only q must be close to nd but also $n$ must be large. For exemple, from Table 6 (when $d=6)$, the Toeplitz algorithm is more efficient only when $n>5$. Notice the great amount of steps that are necessary in these latter cases.

\section{General conclusion}

From the results of Tests 3 and 4, where we considered the extreme cases, we can say as a general conclusion that the type of matrices for which the pencil algorithm is more efficient than the Toeplitz algorithm are matrices with a small degree $d$ but with a structure that implies a number of steps $q$ close to $q_{\max }$, namely matrices with zeros at infinity of high degree. Also notice that in general $n$ must be large. On the other hand, the type of favorable matrices for the Toeplitz algorithm are matrices with $d$ large and $q$ small, namely matrices with poles at infinity of high degree.

We have seen that algorithmic complexity of the pencil algorithm depends on dimension $n$, degree $d$ and logically on the number of steps $q$. Then although the matrices with $n$ large, $q$ large and $d$ small are more favorable for the pencil algorithm than for the Toeplitz one, the number of operations performed by both algorithms will be comparatively large. On the other hand, we can easily find a polynomial matrix very favorable for the Toeplitz algorithm and simultaneously very unfavorable for the pencil algorithm, as we can see in the following examples.

Example 1 Consider the $40 \times 40$ polynomial matrix

$$
A(s)=\left[\begin{array}{cccc}
s & & & 0 \\
& 1 & & \\
& & \ddots & \\
0 & & & 1
\end{array}\right]
$$

which has 1 pole at infinity of degree 1 and 39 zeros at infinity of degree 0 . We can see that the vector of nullities is

$$
v=[39,0] \text {. }
$$

The pencil algorithm is more efficient: the number of opera-

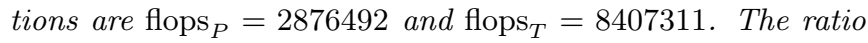
between this number of operations is

$$
\frac{\text { flops }_{T}}{\text { flops }_{P}}=2.93 .
$$

Now consider the $40 \times 40$ polynomial matrix

$$
A(s)=\left[\begin{array}{cccc}
1 & s^{2} & & 0 \\
& \ddots & \ddots & \\
& & \ddots & s^{2} \\
0 & & & 1
\end{array}\right]
$$

which has 39 poles at infinity of degree 2 and 1 zero at infinity of degree 78. The vector of nullities is

$$
v=[\underbrace{1,1, \ldots, 1}_{80}, 0] \text {. }
$$

The pencil algorithm is more efficient: the number of operations are flops $_{P}=324244800$ and flops $_{T}=2052129600$. The ratio between this number of operations is

$$
\frac{\text { flops }_{T}}{\text { flops }_{P}}=6.33 \text {. }
$$

In this example we can notice that although the pencil algorithm is more efficient, the number of operations that both algorithm perform is very large. Also notice that the ratios are not very large.

Example 2 Consider the matrix

$$
A(s)=\left[\begin{array}{lll}
s^{40} & & \\
& s^{39} & \\
& & s^{39}
\end{array}\right]
$$


which has 1 pole at infinity of degree 40 and 2 poles at infinity of degree 39. the vector of nullities is

$$
v=[2,0] .
$$

The Toeplitz algorithm is more efficient: the number of operations are flops $P_{P}=87946704$ and flops $_{T}=2502$. The ratio between this number of operations is

$$
\frac{\text { flops }_{P}}{\operatorname{flops}_{T}}=35150.56 \text {. }
$$

In this example we can see the great difference between the number of operations performed by the pencil algorithm and the Toeplitz algorithm.

\section{Conclusions}

In this paper we have improved the Toeplitz algorithm originally proposed in [5] to compute infinite structural indices of polynomial matrices. By taking advantage of the special block Toeplitz structure of the problem we have reduced the number of operations involved in the computation. A bunch of numerical experiments indicate that the Toeplitz algorithm can be considered as an alternative, if not a competitor, to the classical pencil matrix algorithm originally proposed in [13].

Our work can be extended in various directions:

- It would be insightful to carry out a backward error anaysis (see e.g. [9] for a nice introduction) of the Toeplitz algorithm and to compare the obtained error bounds with those of the pencil algorithm.

- With the Toeplitz algorithm the whole infinite eigenstructure (structural indices + eigenvectors) of the polynomial matrix can be obtained with almost no extra computational effort. We are not aware of any straightforward modification of the pencil algorithm to obtain the eigenstructure of a polynomial matrix. It is not obvious to us how the infinite eigenstructure of a polynomial matrix relates with that of its associated matrix pencil. Besides structural indices, eigenvectors are also important when solving several problems such as computing the subspace of impulsive solutions of linear homogeneous matrix differential equations, or extracting infinite zeros of a polynomial matrix to improve the computation of finite zeros, see [5] and references therein.

- The Toeplitz algorithm as presented in this paper can also pave the way for the development of more general structured algorithms dealing with medium-size or large polynomial matrices. We are currently studying the application of block Toeplitz algorithms to extract minimal bases for polynomial null-spaces of rank deficient polynomial matrices. It is likely that similar algorithms can be used to solve large polynomial matrix Diophantine equations arising in pole placement problems. Relationships with the block Toeplitz algorithms recently described in [7] must also be clarified.
- Finally, we plan to apply the Toeplitz algorithm to perform multivariable decoupling and solve related control problems, along the direction reported in our companion paper [16].

\section{Acknowledgments}

Juan-Carlos Zúñiga acknowledges support from the National Council of Science and Technology of Mexico (CONACYT) and from the Secretariat of public education of Mexico (SEP). Didier Henrion acknowledges support from the Grant Agency of the Czech Republic under Project No. 102/02/0709.

\section{References}

[1] Callier F.M. and Desoer C.A., Multivariable Feedback Systems. Springer Verlag, Berlin, 1982.

[2] Golub G.H. and Van Loan C.F., Matrix Computations. The Johns Hopkins University Press, New York, 1996.

[3] Gantmacher F.R., Matrix Theory. Chelsea Publishing, New York, 1959.

[4] Henrion D. and Šebek M., An Algorithm for Polynomial Matrix Factor Extraction. International Journal of Control, Vol. 73, No. 8, pp. 686-695, 2000.

[5] Henrion D., Ruiz-León. J.J. and Šebek. M., Extraction of Infinite Zeros of Polynomial Matrices. Proceedings of the IEEE Conference on Decision and Control, pp. 42214226, Sydney, Australia, 2000.

[6] Kailath T., Linear Systems. Prentice Hall, Englewood Cliffs, 1980.

[7] Kressner D. and Van Dooren P., Factorizations and linear system solvers for matrices with Toeplitz structure. Slicot Working Note, No. 2000-2, Katholieke Universiteit Leuven, Belgium, 2001.

[8] Kučera V., Discrete Linear Control: The Polynomial Equation Approach. John Wiley and Sons, Chichester, 1979.

[9] Petkov P.Hr., Christov N.D. and Konstantinov M.M.,. Computational Methods for Linear Control Systems. Prentice Hall, New York, 1991.

[10] Polyx, Ltd. The Polynomial Toolbox for Matlab. Version 2.5 released in 2000. See www. polyx.cz

[11] Slicot: Control and Systems Library, Working Group on Software, Katholieke Universiteit Leuven, Belgium, 2001. See www.win.tue.nl/niconet

[12] Van Dooren P., Dewilde P. and Vandewalle J., On the Determination of the Smith-MacMillan Form of a Rational Matrix from its Laurent Expansion. IEEE Transactions on Circuits and Systems, Vol. 26, No. 3, pp. 180-189, 1979.

[13] Van Dooren P., The Computation of Kronecker's Canonical Form of a Singular Pencil. Linear Algebra and Its Applications, Vol. 27, pp. 103-141, 1979.

[14] Van Dooren P and Dewilde P., The Eigenstructure of an Arbitrary Polynomial Matrix. Computational Aspects. Linear Algebra and Its Applications, Vol. 50, pp. 545$580,1983$. 
[15] Vardulakis A.I.G., Linear Multivariable Control. Algebraic Analysis and Synthesis Methods. Wiley, Chichester, 1991.

[16] Zúñiga J.C., Ruiz-León J.J. and Henrion D., Algorithm for decoupling and pole assignment of linear multivariable systems. Research Report, LAAS-CNRS, Toulouse, France, 2002. Submitted to the European Control Conference, 2003. 